\title{
SIRT1 promotes DNA repair activity and deacetylation of Ku70
}

\author{
Jaemin Jeong ${ }^{1,2}$, Kyungmi Juhn ${ }^{1}$, \\ Hansoo Lee ${ }^{2}$, Sang-Hoon $\mathrm{Kim}^{3}$, \\ Bon-Hong Min ${ }^{4}$, Kyung-Mi Lee ${ }^{5}$, \\ Myung-Haeng Cho ${ }^{6}$, Gil-Hong Park ${ }^{5,7}$ \\ and Kee-Ho Lee ${ }^{1,7}$
}

\author{
${ }^{1}$ Laboratory of Molecular Oncology \\ Korea Institute of Radiological and Medical Sciences \\ Seoul 139-706, Korea \\ ${ }^{2}$ Vascular System Research Center \\ Division of Life Sciences \\ College of Natural Sciences \\ Kangwon National University \\ Chunchon 200-701, Korea \\ ${ }^{3}$ Department of Biology \\ Research Institute for Basic Sciences \\ Kyung Hee University \\ Seoul 130-701, Korea \\ ${ }^{4}$ Department of Pharmacology and \\ BK21 Program for Medical Sciences \\ ${ }^{5}$ Department of Biochemistry and \\ Division of BK 21 Program for Biomedical Science \\ Korea University College of Medicine \\ Seoul 136-701, Korea \\ ${ }^{6}$ Laboratory of Toxicology \\ College of Veterinary Medicine \\ Seoul National University \\ Seoul 151-742, Korea \\ ${ }^{7}$ Corresponding authors: Tel, 82-2-970-1313; Fax, 82-2-970-2402; \\ E-mail, khlee@kcch.re.kr (for K.H.L.) and \\ Tel, 82-2-920-6182; Fax, 82-2-928-4853; \\ E-mail, ghpark@korea.ac.kr (for G.H.P.)
}

Accepted 9 November 2006

Abbreviations: HDAC, histone deacetylase; SIR2, silent information regulator 2

\begin{abstract}
Human SIRT1 controls various physiological responses including cell fate, stress, and aging, through deacetylation of its specific substrate protein. In processing DNA damage signaling, SIRT1 attenuates a cellular apoptotic response by deacetylation of $\mathrm{p} 53$ tumor suppressor. The present study shows that, upon exposure to radiation, SIRT1 could enhance DNA repair capacity and deacetylation of repair protein Ku70. Ectopically over-expressed
\end{abstract}

\begin{abstract}
SIRT1 resulted in the increase of repair of DNA strand breakages produced by radiation. On the other hand, repression of endogenous SIRT1 expression by SIRT1 siRNA led to the decrease of this repair activity, indicating that SIRT1 can regulate DNA repair capacity of cells with DNA strand breaks. In addition, we found that SIRT1 physically complexed with repair protein Ku70, leading to subsequent deace tylation. The dominant-negative SIRT1, a catalytically inactive form, did not induce deacetylation of Ku70 protein as well as increase of DNA repair capacity. These observations suggest that SIRT1 modulates DNA repair activity, which could be regulated by the acetylation status of repair protein Ku70 following DNA damage.
\end{abstract}

Keywords: DNA damage; DNA repair; Ku autoantigen; radiation, ionizing; SIRT1 protein, human

\section{Introduction}

Members of the silent information regulator (SIR2) gene family are highly conserved, ranging from archaebacteria to eukaryotes (Frye, 2000). The Sir2 protein functions as a NAD-dependent protein deacetylase and is thought to mediate silencing by regulating histone acetylation (Imai et al., 2000; Landry et al., 2000; Smith et al., 2000). In an enzymatic reaction, Sir2 catalyzes the elimination of an acetyl group from the lysine residue of substrate proteins (Moazed, 2001). The enzymatic activity of Sir2 proteins is mainly exerted by its core domain containing 260 amino acids that is conserved among Sir2 family genes (Sherman et al., 1999), and mutations of the conserved residues within this globular core domain leads to a loss of deacetylase acivity (Frye, 1999; Sherman et al., 1999; Tanny et al., 1999). At least three of the SIR proteins in yeast, Sir2, 3 and 4, participate in gene silencing, as well as in proper cell cycle progression, radiation resistance and genomic stability (Brachmann et al., 1995; Shore, 2000; Gasser and Cockell, 2001).

Human has seven proteins with homology to Sir2, called as sirtuins (Frye, 2000), however their roles are poorly understood. Recently, it has been found that the human homologue of Sir2, SIRT1, binds to and regulates p53 tumor suppressor (Luo et al., 2001; Vaziri et al., 2001; Brunet et al., 2004; Cohen et al., 2004b): Following DNA damage response, SIRT1 deacetylates p53 protein and attenuates p53 protein's ability as a transcription factor. Conse- 
quently, SIRT1 over-expression increases cell survival under DNA damage inducing conditions (Chen et al., 2005). These earlier observations suggest a possibility that signals generated during the process of DNA repair are delivered via SIRT1 to acetylated p53. We present herein functional evidence for the involvement of SIRT1 in DNA repair response to radiation. In addition, this modulation of DNA repair activity may be connected to deacetylation of Ku70 proteins.

\section{Materials and Methods}

\section{Cell culture}

Q293A cells (Quantum Biotech., Montreal, Canada) were maintained in Dulbecco's modified eagle's medium (DMEM) supplemented with $5 \%$ fetal bovine serum. The cells were maintained in $37^{\circ} \mathrm{C}$ humidified atmosphere incubator with $5 \% \mathrm{CO}_{2}$.

\section{Retrovirus production and infection}

Human SIRT1 and SIRT1-HY cDNA (Luo et al., 2001; Vaziri et al., 2001) was subcloned into the retroviral vector pMFG-puro. The retroviruses were produced by transient transfection of pMFG-puro, MFG-SIRT1 and MFG-SIRT1-HY (deacetylase dead mutant) plasmids into $\mathrm{H} 29 \mathrm{D}$ packaging cells. Q293A cells were infected with retrovirus containing $8 \mu \mathrm{g} / \mathrm{ml}$ of polybrene for $4 \mathrm{~h}$. Twenty four hours after the infection, the cells were selected in $2 \mu \mathrm{g} / \mathrm{ml}$ puromycin (BD Biosciences, Palo Alto, CA).

\section{Immunoprecipitation and Western blot analysis}

The cells were lysed in a TNN buffer $(120 \mathrm{mM} \mathrm{NaCl}$, $40 \mathrm{mM}$ Tris- $\mathrm{HCl}, \mathrm{pH} 8.0,0.5 \% \mathrm{NP}-40,1 \mathrm{mM}$ phenylmethylsulphonyl fluoride, $1 \mathrm{mM}$ sodium orthovanadate, $100 \mathrm{mM}$ sodium fluoride and $1 \mu \mathrm{g} / \mathrm{ml}$ each of leupeptin, aprotinin and pepstatin). Immunoprecipitates were boiled in SDS-sample buffer, loaded on $7.5 \%$ SDS-polyacrylamide gels and separated by electrophoresis. Proteins were electrophoretically transferred to PROTRAN Nitrocellulose Transfer Membrane (Schleicher \& Schuell, Germany). Detection of primary antibodies was performed using horseradish peroxidase-conjugated donkey anti-rabit IgG or anti-goat IgG using Luminal Reagent (Santa Cruz, CA).

\section{Measurement of DNA repair capacity}

The CMV promoter was subcloned into pGL2 plasmid carrying a firefly luciferase gene (Promega) as described previously (Zeng et al., 1999). This plasmid, termed pGL2-CMV, was used to determine DNA repair capacity. Thus, pGL2-CMV plasmid was da- maged in vitro by exposure to $\gamma$-ray using a ${ }^{137} \mathrm{Cs}$ source. pMFG-SIRT1 was transiently co-transfected into Q293A cells with damaged or un-damaged pGL2-CMV and pCH110 (Pharmacia, Cleveland, Ohio) using calcium phosphate precipitation method (Invitrogen, Carlsbad, CA). The cells were collected $72 \mathrm{~h}$ after the transfection, and the cell extracts were used to assay luciferase activity. The protein concentrations were determined using a Bio-Rad protein assay kit. The lac activity induced by $\mathrm{pCH} 110$ was determined in $72 \mathrm{~h}$ by using orthonitrophenyl- $\beta$ D-galgactopyranoside as a substrate. The luciferase activity was determined with a Promega luciferase assay system and represented as relative luciferase units by normalizing to a cotransfected lacZ control acivity in each transfection.

\section{SIRT1 siRNAs}

The sequences of sense and anti-sense RNA were as follows: pJJ71 5'-AACTTGTACGACGAAGACGAC-3', pJJ72 5'-AAAGTGATGAGGAGGATAGAG-3', pJJ73 5'-AATTCCAGCCATCTCTCTGTC-3', pJJ75 5'-AACAGTTTCATAGAGCCATGA-3', pJJ76 5'-AACCTTTGCCTCATCTGCATT-3', pJJ77 5'-AACTTCACCACCAGATTCTTC-3' for the SIRT1 gene. These SIRT1-siRNA oligonucleotides were subcloned into siRNA construction vector pSilencer-neo2 according to manufacturer's instructions (Ambion, Austin, TX). Q293A cells were transfected with these SIRT1 siRNA plasmids using calcium phosphate precipitation method. The cells were collected $48 \mathrm{~h}$ after the transfection, and the cell extracts were used to confirm the effect of siRNA by Western blot analysis.

\section{Results}

\section{Enhancement of DNA repair capacity by SIRT1}

Recent studies have provided evidences that SIRT1 increases cell survival upon stress response including hydrogen peroxide (Luo et al., 2001), anticancer drugs (Luo et al., 2001), and ionizing radiation (Vaziri et al., 2001). To further evaluate the mechanistic basis of SIRT1 in increasing cell survival under DNA damage inducing condition, we examined whether SIRT1 could modulate DNA repair capacity which could critically affect the sensitivity to anticancer agents including radiation. To monitor DNA repair activity, we analyzed cell's ability to repair plasmid DNAs with strand breaks. Either SIRT1 or empty control vector was cotransfected into Q293A cells with the luciferase reporter plasmid which had been broken by various doses of irradiation prior to transfection, and the capacity to repair broken plasmid was then determined by measuring the luciferase activity. Introduction of SIRT1 resulted 


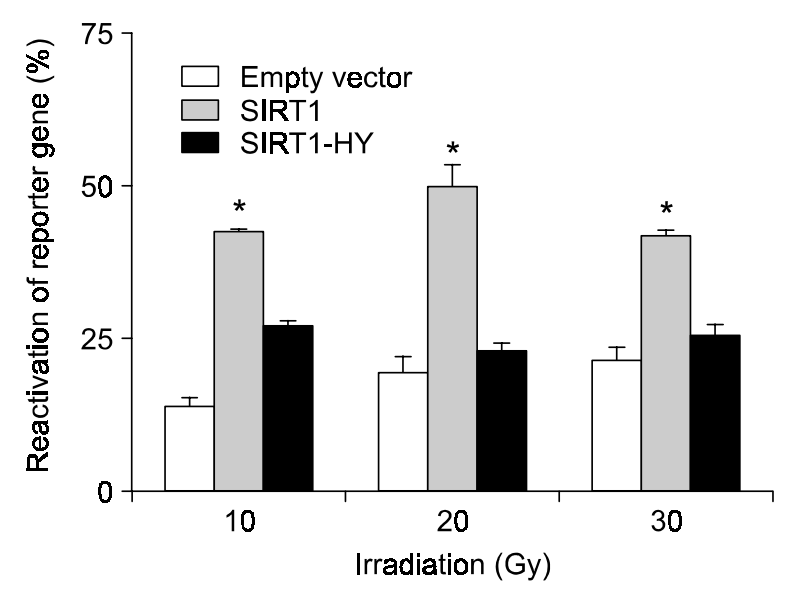

Figure 1. SIRT1-mediated increase of healing DNA breaks produced by radiation. Q293A cells were cotransfected with either SIRT1 (gray bar), SIRT1-HY, a dominant-negative SIRT1 (black bar), or empty vector control (white bar), and reporter plasmids were broken by various doses of irradiation. Seventy two hours after the transfection, rejoining capacity of the breakage sites was determined by measuring the luciferase activity as described in Materials and Methods. The values are represented as a mean from three separate experiments; error bars, \pm SD. ${ }^{*} P<0.05$ SIRT1 versus empty vector.

in the increase of relative luciferase activity compared to that of MFG empty control vector (Figure 1), indicating the increase of DNA repair activity by ectopically over-expressed SIRT1. Specifically, when breakages of luciferase reporter plasmid were generated by exposure to 10,20 , and 30 Gy radiation prior to transfection, SIRT1 led to 2.6, 3.3, and 2.4 fold increases in luciferase activities, respectively, compared to those of empty vector control.

\section{Repression of DNA repair capacity by SIRT1 siRNA}

The above result was obtained by ectopically overexpressed SIRT1. Therefore, it was felt necessary to monitor DNA repair activity, when endogenous level of SIRT1 was lowered. Therefore, we applied a siRNA technology to reduce endogenous SIRT1. In order to get the most efficient siRNA to SIRT1, we designed 6 different siRNAs within the expression loci of SIRT1 gene and then co-transfected them into Q293A cells with SIRT1 expression plasmid. Figure $2 A$ shows that all these siRNA efficiently reduced the level of exogenously overexpressed SIRT1 protein. Therefore, we selected one SIRT1 siRNA which lowered the SIRT1 protein level most effectively (Figure 2A). The selected SIRT1 siRNA also led to the decrease of endogenous as well as exogenous SIRT1 protein (Figure 2B). Q293A cells transfected with SIRT1 siRNA exhibited a marked reduction of DNA repair capacity, compared to those transfected with wild-type SIRT1. In contrast to wild type SIRT1,
A

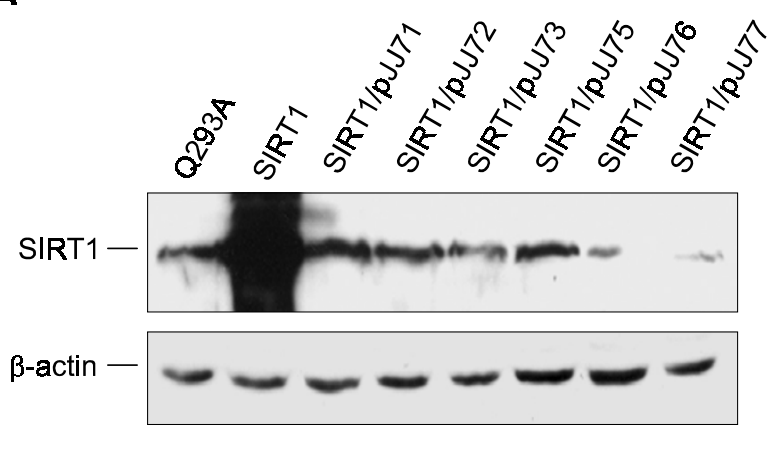

B

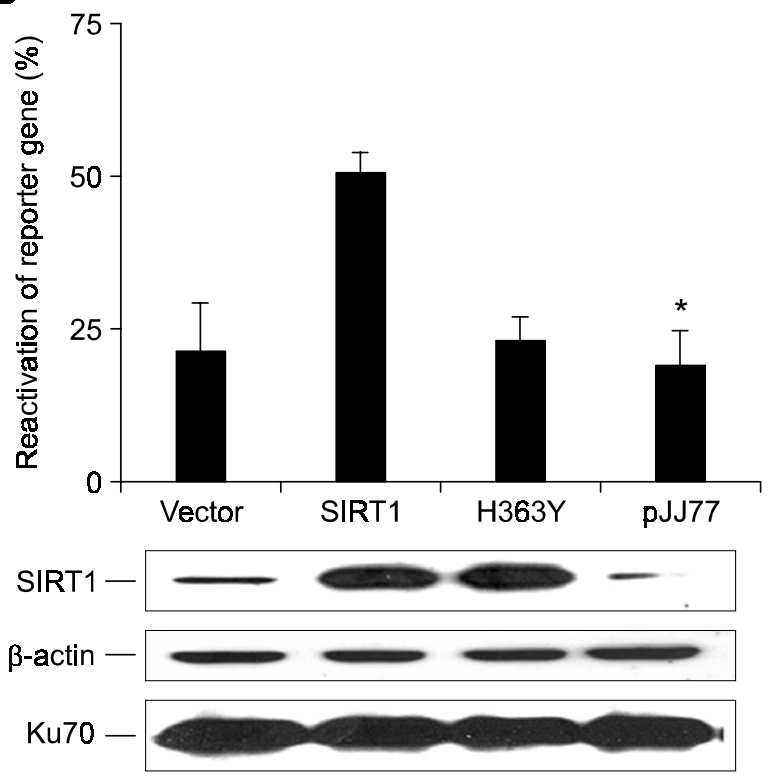

Figure 2. Reduction of DNA repair activity by SIRT1-SiRNA. Q293A cells were cotransfected with SIRT1, and the reduction power of six SIRT1 siRNAs prepared by pSilencer kit, on SIRT1 expression was determined by Western blot analysis. SIRT1/pJJ77 with the most active SIRT1 siRNA in reducing SIRT1 expression (A) was co-transfected with SIRT1 expression plasmid into Q293A cells and the repair activity was tested, as described in the legend of Figure 1. $\beta$-actin was used as an internal loading control. The values are represented a mean from three separate experiments; error bars, $\pm \mathrm{SD}$. * $P<0.05$ SIRT1 versus pJJ77.

dominant-negative SIRT1, SIRT1-HY which expresses catalytically inactive form mutated at residue 363 histidine of SIRT1 protein by replacing with tyrosine (Vaziri et al., 2001), failed to enhance DNA repair capacity. These findings indicate that SIRT1 protein can enhance DNA repair capacity in vivo following DNA damage.

\section{Interaction of SIRT1 with Ku70}

The result that SIRT1, a known deacetylase, could 
A

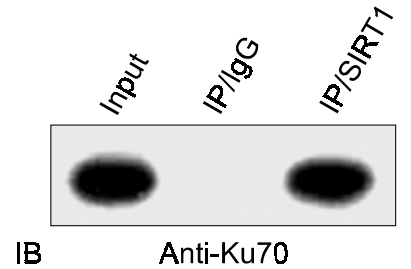

B

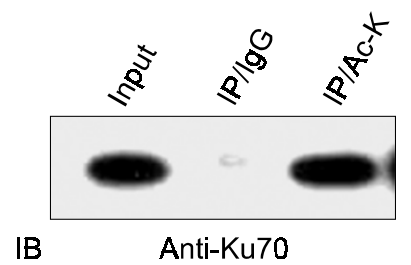

C

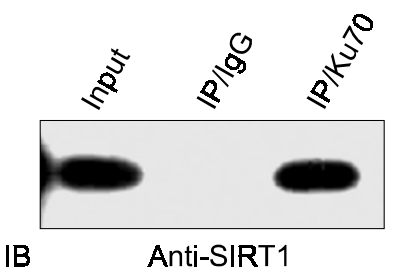

D

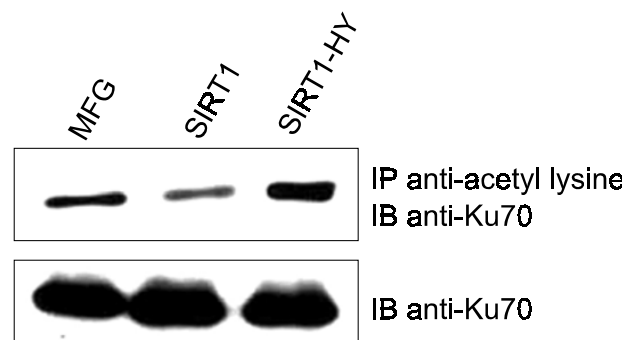

E

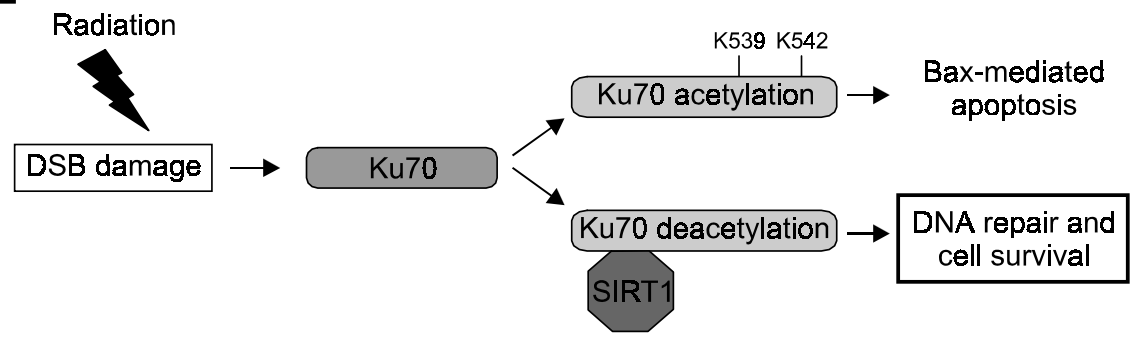

Figure 3. Binding of SIRT1 and subsequent deacetylation of Ku70 protein. Q293A cells were transiently transfected with SIRT1 and lysed $36 \mathrm{~h}$ later. The cell lystes were immunoprecipitated with anti-SIRT1 (A), anti-Ku70 antibody (B), or control IgG antibody, and then reciprocally probed with anti-Ku70 $(A)$ or anti-SIRT1 antibody (B), respectively. (C) For the analysis of acetylation status, the cell lysates were immunoprecipitated using antibody against acetylated-lysine and then probed using anti-Ku70 antibody. (D) Q293A cells were transiently transfected with either SIRT1, SIRT1-HY, or empty vector control plasmid. The cell lyzates were immunoprecipitated using anti-acetylated-lysine (upper panel) or anti-Ku70 antibody (lower panel), respectively, and then Western blotted for detection of Ku70 protein. (E) Proposed model for functional modulation of repair protein Ku70 by SIRT1. Acetylation status of repair protein Ku70 may regulate cell's fate under DNA damage inducing condition: Ku70 acetylation by CBP or PCAF accelerates cell death (Cohen et al., 2004a), whereas its deacetylation by SIRT1 promotes cell survival via increase of DNA repair activity. modulate DNA repair capacity prompted us to search possible substrate of SIRT1 engaged in DNA repair process. Among several candidate proteins co-localized with SIRT1 at chromosomal end telomere repeats, Ku70 functions as a stimulator of DNA repair protein (Ouyang et al., 1997) and has been shown to be recruited with SIRT1 to the sites of broken chromosome (Hegde and Klein, 2000). Therefore, we examined whether SIRT1 could physically form complex with Ku70. Since low level of endogenous SIRT1 would make it difficult to examine its binding to cellular substrate, we prepared SIRT1 overexpressing cells. Thus, we infected U2OS cells with retrovirus carrying SIRT1 and then isolated mixed populations resistant to puromycine. The mixed populations exhibited high level of exogenous SIRT1 and endogenous Ku70 expressions (data not shown), and we then examined possible interactions between SIRT1 and Ku70 proteins in the lysate of U2OS cells. These cell lysates were subsequently immuno-precipitated with anti-SIRT1 antibody and the resulting immune complexes were analyzed by immunoblotting with antiKu70 antibody. As shown in Figure 3A, immuno- precipitation of SIRT1 from the lysates of U2OS cells etopically overexpressing SIRT1 resulted in coimmunoprecipitation of Ku70. This interaction was reciprocally further confirmed by immunoprecipitating the lysate with anit-Ku70 antibody and subsequently probing the blotted precipitate with SIRT1 antibody (Figure 3B). On the other hand, we could not detect Ku70 or SIRT1 protein in the immunoprecipitation of $\lg G$ control antibody from the same cell lysates. These findings indicate that SIRT1 and Ku70 can interact and physically form complex with one another in vivo.

\section{Deacetylation of Ku70 by SIRT1}

The above result that SIRT1, a known deacetylase, can physically form complex with Ku70 led us to examine whether SIRT1 could deacetylase Ku70 in vivo. As recently reported in 293 cell system by (Cohen et al., 2004a) Ku70 acetylation in U2OS cells was confirmed by showing its presence in the acetylated protein pools immunoprecipitated by antiacetyl-lysine antibody (Figure 3C) (Cohen et al., $2004 b)$. This acetylated form of Ku70 protein co- 
immunoprecipitated by anti-acetyl lysine antibody was reduced by ectopically over- expressed SIRT1 (Figure 3D). The introduction of dominant negative SIRT1, SIRT1-HY, however, resulted in the restoration of the level of the acetylated Ku70 protein, and Western blot assay by Ku70 antibody revealed we could not find detectable changes of total Ku70 protein. Therefore, the present observation provides evidence that SIRT1 can deacetylate Ku70 protein in vivo by physically forming complexes with one another. In addition, our present finding together with recent report that acetylation of repair protein Ku70 accelerates cell death upon exposure to genotoxic agents (Cohen et al., 2004b) suggest that SIRT1 may increase DNA repair capacity via deacetylation of SIRT1 protein (Figure 3E).

\section{Discussion}

In response to DNA damage, SIRT1 protein binds to and deacetylates specific lysine residue of substrate proteins, the modification of which leads to the repression of their transcriptional activities, resulting in the promotion of cell survival (Luo et al., 2001; Vaziri et al., 2001; Motta et al., 2004; Picard et al., 2004). SIRT1, therefore, has been suggested to be a suppressor of apoptotic responses (Luo et al., 2001; Vaziri et al., 2001). Related to the DNA damage pathway, our present study demonstrated that, when exposed to radiation, SIRT1 enhanced DNA repair activity and physically formed complexes with repair protein Ku70, and subsequently deacetylated the latter; This scenario could be one plausible mechanistic basis of the promotion of cell survival.

In yeast, both Ku70 and Sir2, yeast homologue of mammalian SIRT1 protein, associate at the telomere and are recruited to the cleavage site induced by DNA breaks inducing agents (Martin et al., 1999). Although the involvement of Ku70 in repairing DNA double-strand break has well been verified in various systems, it is still unclear how Ku70 is modulated and modified in response to DNA damage. Therefore, we examined whether SIRT1 might regulate DNA repair capacity through modulation of Ku70 activity. As expected, SIRT1 protein directly interacted with Ku70 protein to physically form complex, and this interaction might possibly control acetylation status of Ku70 protein. Indeed, the formation of SIRT1-Ku70 complex resulted in an enhancement of rejoining broken DNA. Therefore, our present finding on deacetylation of Ku70 by SIRT1 and resulting increase of repair activity directly gives support to a recent observation that Ku70 protein is acetylated by several acetyl-transferases such as p300, PCAF, and $\mathrm{CBP}$, and that this acetylation process accele- rates Bax-mediated apoptosis (Cohen et al., 2004a). This contention further suggests that, upon DNA damage, SIRT1 links to the modulation of repair activity via acetylation-deacetylation cycle of Ku70 protein and consequently promotes cell survival.

It has recently, been uncovered that SIRT1 is critically involved in life span extension by calorie restriction which shifts metabolic pathways (Anderson et al., 2003; Cohen et al., 2004b; Motta et al., 2004). The metabolic switches regulated by SIRT1 are PGC1- $\alpha$ (Rodgers et al., 2005), PPAR- $\gamma$ (Picard et al., 2004), and FOXO3a (Motta et al., 2004; Nemoto et al., 2004) that control glucose and fatty acid metabolisms. In addition to these metabolic switches, the decrease of stress-induced apoptotic cell death via deacetylation of repair protein Ku70 was proposed to contribute to the longevity by calorie restriction (Cohen et al., 2004b). As assessed above, our present study demonstrated that SIRT1 increased the repair activity of cells exposed to radiation. Since the enhanced repair capacity was reduced below its basal level when SIRT1 siRNA was transduced into the cells overexpressing SIRT1, our present result on Ku70 modulation by SIRT1 strongly suggests a possibility that the regulation of acetylation status of Ku70 protein might be one mechanistic clue of life span extension by calorie restriction. The present results together with other relevant studies further suggest that the formation of SIRT1-Ku70 complex has a profound effect on overcoming hazardous changes of environment which are produced by stresses, such as DNA breakage, thereby extending life span.

Until now, SIRT1 has been shown to deacetylate various substrate proteins involved in DNA damage response, including p53 (Luo et al., 2001; Vaziri et al., 2001), FOXO (Brunet et al., 2004), NF-אB (Yeung et al., 2004), as well as Ku70, suggesting that SIRT1 interconnects various pathways responding to DNA damage by deacetylating the above described substrates. To better understand the mechanistic and metabolic connections of SIRT1 to DNA damage response, however, more precise coupling between these substrates should be defined: How consecutive cycles of acetylation and deacetylation between them are modulated and whether these connections have any impact on physiological behavior at the level of cell and organism.

\section{Acknowledgement}

We thank that Robert A. Weinberg for providing the SIRT1 and SIRT1-HY plasmids, Prof. Woon Ki Paik for critical reading of the manuscript, and Eun-Ju Lee and Yong-Ho Ham for assistance in the preparation of the manuscript. This work was supported by grants from National Nuclear 
$R$ \& D program and Basic Atomic Energy Research Institute, Korean Ministry of Science and Technology. Work in the lab of K. H. L. was supported by grant from Human Genome Project, MOST. M. H. C. thanks to the support from Korea Research Foundation grant funded by the Korean Government (KRF-2006-311- E00507).

\section{References}

Anderson RM, Latorre-Esteves M, Neves AR, Lavu S, Medvedik O, Taylor C, Howitz KT, Santos H, Sinclair DA. Yeast life-span extension by calorie restriction is independent of NAD fluctuation. Science 2003;302:2124-6

Brachmann CB, Sherman JM, Devine SE, Cameron EE, Pillus L, Boeke JD. The SIR2 gene family, conserved from bacteria to humans, functions in silencing, cell cycle progression, and chromosome stability. Genes Dev 1995;9:2888-902

Brunet A, Sweeney LB, Sturgill JF, Chua KF, Greer PL, Lin Y, Tran H, Ross SE, Mostoslavsky R, Cohen HY, Hu LS, Cheng HL, Jedrychowski MP, Gygi SP, Sinclair DA, Alt FW, Greenberg ME. Stress-dependent regulation of FOXO transcription factors by the SIRT1 deacetylase. Science 2004;303:2011-5

Chen WY, Wang DH, Yen RC, Luo J, Gu W, Baylin SB. Tumor Suppressor HIC1 directly regulates SIRT1 to modulate p53-dependent DNA-damage responses. Cell 2005;123: 437-48

Cohen HY, Lavu S, Bitterman KJ, Hekking B, Imahiyerobo TA, Miller C, Frye R, Ploegh $H$, Kessler BM, Sinclair DA. Acetylation of the $\mathrm{C}$ terminus of Ku70 by CBP and PCAF controls Bax-mediated apoptosis. Mol Cell 2004a;13:627-38

Cohen HY, Miller C, Bitterman KJ, Wall NR, Hekking B, Kessler B, Howitz KT, Gorospe M, de Cabo R, Sinclair DA. Calorie restriction promotes mammalian cell survival by inducing the SIRT1 deacetylase. Science 2004b;305:390-2

Frye RA. Characterization of five human cDNAs with homology to the yeast SIR2 gene: Sir2-like proteins (sirtuins) metabolize NAD and may have protein ADP-ribosyltransferase activity. Biochem Biophys Res Commun 1999;260: 273-9

Frye RA. Phylogenetic classification of prokaryotic and eukaryotic Sir2-like proteins. Biochem Biophys Res Commun 2000;273:793-8

Gasser SM, Cockell MM. The molecular biology of the SIR proteins. Gene 2001;279:1-16

Hegde V, Klein H. Requirement for the SRS2 DNA helicase gene in non-homologous end joining in yeast. Nucleic Acids Res 2000;28:2779-83

Imai S, Armstrong CM, Kaeberlein M, Guarente L. Transcriptional silencing and longevity protein Sir2 is an NADdependent histone deacetylase. Nature 2000;403:795-800

Landry J, Sutton A, Tafrov ST, Heller RC, Stebbins J, Pillus L, Sternglanz R. The silencing protein SIR2 and its homologs are NAD-dependent protein deacetylases. Proc Natl Acad Sci USA 2000;97:5807-11
Luo J, Nikolaev AY, Imai S, Chen D, Su F, Shiloh A, Guarente L, Gu W. Negative control of $p 53$ by Sir2alpha promotes cell survival under stress. Cell 2001;107:137-48

Martin SG, Laroche T, Suka N, Grunstein M, Gasser SM. Relocalization of telomeric Ku and SIR proteins in response to DNA strand breaks in yeast. Cell 1999;97:621-33

Moazed D. Enzymatic activities of Sir2 and chromatin silencing. Curr Opin Cell Biol 2001;13:232-8

Motta MC, Divecha N, Lemieux M, Kamel C, Chen D, Gu W, Bultsma Y, McBurney M, Guarente L. Mammalian SIRT1 represses forkhead transcription factors. Cell 2004;116: 551-63

Nemoto S, Fergusson MM, Finkel T. Nutrient availability regulates SIRT1 through a forkhead-dependent pathway. Science 2004;306:2105-8

Ouyang $\mathrm{H}$, Nussenzweig A, Kurimasa A, Soares VC, Li X, Cordon-Cardo C, Li W, Cheong N, Nussenzweig M, Iliakis G, Chen DJ, Li GC. Ku70 is required for DNA repair but not for T cell antigen receptor gene recombination in vivo. J Exp Med 1997;186:921-9

Picard F, Kurtev M, Chung N, Topark-Ngarm A, Senawong T, Machado De Oliveira R, Leid M, McBurney MW, Guarente L. Sirt1 promotes fat mobilization in white adipocytes by repressing PPAR-gamma. Nature 2004;429:771-6

Rodgers JT, Lerin C, Haas W, Gygi SP, Spiegelman BM, Puigserver P. Nutrient control of glucose homeostasis through a complex of PGC-1alpha and SIRT1. Nature 2005;434:113-8

Sherman JM, Stone EM, Freeman-Cook LL, Brachmann CB, Boeke JD, Pillus L. The conserved core of a human SIR2 homologue functions in yeast silencing. Mol Biol Cell 1999;10:3045-59

Shore $D$. The Sir2 protein family: A novel deacetylase for gene silencing and more. Proc Natl Acad Sci USA 2000;97:14030-2

Smith JS, Brachmann CB, Celic I, Kenna MA, Muhammad S, Starai VJ, Avalos JL, Escalante-Semerena JC, Grubmeyer C, Wolberger C, Boeke JD. A phylogenetically conserved $\mathrm{NAD}^{+}$-dependent protein deacetylase activity in the Sir2 protein family. Proc Natl Acad Sci USA 2000;97:6658-63

Tanny JC, Dowd GJ, Huang J, Hilz H, Moazed D. An enzymatic activity in the yeast Sir2 protein that is essential for gene silencing. Cell 1999;99:735-45

Vaziri H, Dessain SK, Ng Eaton E, Imai SI, Frye RA, Pandita TK, Guarente L, Weinberg RA. hSIR2 (SIRT1) functions as an NAD-dependent p53 deacetylase. Cell 2001;107:149-59

Yeung F, Hoberg JE, Ramsey CS, Keller MD, Jones DR, Frye RA, Mayo MW. Modulation of NF-kappaB-dependent transcription and cell survival by the SIRT1 deacetylase. EMBO J 2004;23:2369-80

Zeng L, Sarasin A, Mezzina M. Novel complementation assays for DNA repair-deficient cells. Transient and stable expression of DNA repair genes. Methods Mol Biol 1999; 113:87-100 